\title{
Atuação dos enfermeiros no acolhimento à demanda espontânea em Estratégia Saúde
}

\section{da Família}

\author{
Nurses' performance in welcome to spontaneous demand in Family Health Strategy \\ Desempeño de las enfermeras en bienvenida a la demanda espontánea en Estrategia de salud \\ Familiar
}

Recebido: 13/01/2022 | Revisado: 17/01/2022 | Aceito: 18/01/2022 | Publicado: 21/01/2022

\author{
Gabriele Zawacki Milagres \\ ORCID: https://orcid.org/0000-0003-3619-9317 \\ Universidade de Santa Cruz do Sul, Brasil \\ E-mail: gabriele93.enf@gmail.com \\ Guilherme Mocelin \\ ORCID: https://orcid.org/0000-0001-9727-3619 \\ Universidade de Santa Cruz do Sul, Brasil \\ E-mail: mocelinguilherme@gmail.com \\ Amanda da Silva de Lima \\ ORCID: https://orcid.org/0000-0001-9788-070X \\ Universidade Católica de Pelotas, Brasil \\ E-mail: amandinha_np4@hotmail.com \\ Gilda Maria de Carvalho Abib El Halal \\ ORCID: https://orcid.org/0000-0002-4709-7212 \\ Universidade Católica de Pelotas, Brasil \\ E-mail: gildaabib@hotmail.com \\ Marcelo Carneiro \\ ORCID: https://orcid.org/0000-0003-3603-1987 \\ Universidade de Santa Cruz do Sul, Brasil \\ E-mail: marceloc@unisc.br \\ Suzane Beatriz Frantz Krug \\ ORCID: https://orcid.org/0000-0002-2820-019X \\ Universidade de Santa Cruz do Sul, Brasil \\ E-mail: skrug@unisc.br
}

\begin{abstract}
Resumo
Introdução: O acolhimento é um recurso destinado a apoiar a qualificação do sistema de saúde, pois possibilita ao usuário, o acesso a um cuidado justo, ampliado e integral, sendo que esse acesso é um direito humano fundamental. Este estudo tem como Objetivo Conhecer a atuação dos enfermeiros no processo de acolhimento à demanda espontânea nas Unidades Básicas de Saúde com Estratégia de Saúde da Família. Metodologia: Trata-se de um estudo exploratório, descritivo com abordagem qualitativa, realizado através de entrevistas semiestruturadas com enfermeiras de unidades básicas. Os dados foram coletados no ano de 2016 e analisados através da Análise de Conteúdo Temática proposta por Bardin. Resultados: Da análise de conteúdo emergiram três categorias: Concepções sobre o acolhimento; Dificuldades e facilidades para a realização do acolhimento; Equipe atuante no acolhimento. Observou-se que, apesar do conhecimento das entrevistadas, elas não conseguem organizar o acolhimento conforme preconizado no seu cotidiano devido às dificuldades elencadas por elas, como a estrutura física e pela grande demanda espontânea. Considerações Finais: Para que o acolhimento seja de qualidade e resolutivo, os profissionais terão que ampliar o momento da escuta, criar vínculos e realizar ações programáticas junto à população para resolver problemas com a demanda reprimida.
\end{abstract}

Palavras-chave: Acolhimento; Enfermeiros; Estratégia de Saúde da Família.

\begin{abstract}
Introduction: Welcoming is a resource designed to support the qualification of the health system, as it provides the user with access to fair, expanded and comprehensive care, and this access is a fundamental human right. This study aims to Understand The role of nurses in the process of welcoming spontaneous demand in Basic Health Units with the Family Health Strategy. Methodology: This is an exploratory, descriptive study with a qualitative approach, carried out through semi-structured interviews with nurses from basic units. Data were collected in 2016 and analyzed through the Thematic Content Analysis proposed by Bardin. Results: From the content analysis, three categories emerged: Conceptions about user embracement; Difficulties and facilities for carrying out the reception; Team active in the reception. It was observed that, despite the interviewees' knowledge, they are unable to organize the reception
\end{abstract}


as recommended in their daily lives due to the difficulties listed by them, such as the physical structure and the large spontaneous demand. Final Considerations: For the reception to be of quality and resolute, professionals will have to expand the listening time, create bonds and carry out programmatic actions with the population to solve problems with the repressed demand.

Keywords: User Embracement; Nurses; Family Health Strategy.

\section{Resumen}

Introducción: La acogida es un recurso diseñado para apoyar la calificación del sistema de salud, ya que brinda al usuario acceso a una atención justa, ampliada e integral, y este acceso es un derecho humano fundamental. Este estudio tiene como Objetivo Comprender el papel del enfermero en el proceso de acogida de la demanda espontánea en las Unidades Básicas de Salud con la Estrategia Salud de la Familia. Metodología: Se trata de un estudio exploratorio, descriptivo con abordaje cualitativo, realizado a través de entrevistas semiestructuradas con enfermeros de unidades básicas. Los datos se recopilaron en 2016 y se analizaron a través del Análisis de contenido temático propuesto por Bardin. Resultados: Del análisis de contenido, surgieron tres categorías: conceptos sobre la aceptación del usuario; Dificultades e instalaciones para llevar a cabo la recepción; Equipo activo en la recepción. Se observó que, a pesar de los conocimientos de los entrevistados, no logran organizar la recepción como recomiendan en su vida diaria debido a las dificultades enumeradas por ellos, como la estructura física y la gran demanda espontánea. Consideraciones Finales: Para que la recepción sea de calidad y resuelta, los profesionales deberán ampliar el tiempo de escucha, crear vínculos y realizar acciones programáticas con la población para solucionar problemas con la demanda reprimida.

Palabras clave: Anfitrión; Enfermeras; Estrategia de Salud Familiar.

\section{Introdução}

A Atenção Primária em Saúde (APS), na perspectiva do Sistema Único de Saúde (SUS), é vista como um conjunto de ações, que tem como um de seus principais focos de atuação promover a saúde dos indivíduos, da família e da comunidade. É a porta de entrada dos usuários ao sistema de saúde, responsável pela coordenação do cuidado e organização do sistema e tem por objetivo orientar, solucionar e direcionar os usuários aos serviços de saúde (Brasil, 2017; Báfica et al., 2021). Assim, uma das grandes referências da APS é o acolhimento prestado ao usuário.

O acolhimento surgiu a partir das discussões sobre a reorganização da assistência. É um recurso destinado a apoiar a qualificação do sistema de saúde, pois possibilita, ao usuário, o acesso a um cuidado justo, ampliado e integral, a partir do reconhecimento de que esse acesso é um direito humano fundamental (Silva et al., 2020). Segundo o Ministério da Saúde (MS) a unidade de saúde precisa acolher e classificar a demanda com o intuito de tornar viável o acesso ao serviço de saúde (Brasil, 2017).

É necessário que os profissionais compreendam o acolhimento visando a um atendimento baseado nos princípios e diretrizes do SUS (Brasil, 1990). Neste sentido, desde o lançamento da Política Nacional de Humanização (PNH), o acolhimento é definido como uma diretriz, que tem o intuito de fazer com que o sistema de saúde seja mais resolutivo e que tenha qualidade na atenção (Brasil, 2004). Deste modo, torna-se um mecanismo primordial da APS, pelo fato de facilitar, dinamizar e organizar o processo de trabalho dos profissionais (Brasil, 2013). Assim, os mesmos devem se disponibilizar a ouvir atentamente a demanda do usuário, compreender as reais necessidades e integrá-las ao conhecimento científico, a fim de garantir resolubilidade e humanização no atendimento (Marques \& Silva, 2020).

Os enfermeiros são profissionais que se destacam cada vez mais no mercado de trabalho por possuírem habilidades aguçadas na compreensão do indivíduo em todas as suas dimensões. Na atuação junto à Estratégia Saúde da Família (ESF), o papel do enfermeiro está diretamente relacionado à prática do acolhimento, na qual busca criar uma relação efetiva com o usuário durante a escuta, unindo os conhecimentos adquiridos durante a formação aos saberes empíricos se aproximando do usuário e identificando suas necessidades e expectativas (Lopes et al., 2020; Morelato et al., 2021). O acolhimento é um dispositivo de humanização potente para reduzir a demanda reprimida e possibilita qualificar o acesso aos serviços (Gusmão et al., 2021), assim sendo, o objetivo desta pesquisa foi conhecer a atuação dos enfermeiros no acolhimento à demanda espontânea nas Unidades Básicas de Saúde (UBS) com ESF. 


\section{Metodologia}

Trata-se de um estudo exploratório e descritivo com abordagem qualitativa (Lacerda et al., 2018; Minayo, 2014). O mesmo foi realizado junto a quatro UBS que são vinculadas a um hospital universitário, localizado na cidade de Pelotas, interior do Rio Grande do Sul. Estas unidades são credenciadas junto ao MS como ESF, tendo oito equipes formadas por Médicos, Enfermeiros, Técnicos de Enfermagem e Agentes Comunitários de Saúde (ACS). Sendo que seis ESF têm ainda como parte da equipe multiprofissional, o Cirurgião-dentista e Auxiliar em Saúde Bucal. Além destes, outros profissionais técnicos e docentes, como Assistentes Sociais e Psicólogos, atuam junto às UBS prestando assistência à população e na formação de profissionais de saúde.

Os sujeitos entrevistados foram todas as enfermeiras que atuam nas UBS escolhidas, no total de oito profissionais. Os dados foram coletados no ano de 2016, através de entrevista semiestruturada, com tempo médio de duração de trinta minutos. Estas foram gravadas em áudio e posteriormente transcritas em arquivos de texto para a realização da análise dos dados.

Os dados foram analisados de acordo com a Análise de Conteúdo Temática proposta por Bardin (Bardin, 2011). O processo de análise do material compreendeu as seguintes fases: a) transcrição das entrevistas e pré-análise; b) exploração do material através da leitura das entrevistas transcritas; c) tratamento dos resultados e interpretação.

O estudo foi autorizado pela instituição e aprovado pelo Comitê de Ética em Pesquisa (Número do Parecer: 1.685.365), além disso, todas as entrevistadas assinaram o Termo de Consentimento Livre e Esclarecido.

\section{Resultados e Discussão}

Os sujeitos que participaram da pesquisa são enfermeiras, todas do sexo feminino, na faixa etária entre vinte e seis a quarenta anos de idade. O tempo de atuação em ESF variou de 3 meses a 3 anos. Duas delas já concluíram pós-graduação em ESF, duas estão com pós-graduação em andamento e quatro relataram não possuir nenhuma especialização.

Para apresentação dos resultados foram estabelecidas três categorias temáticas: Concepções sobre o acolhimento; Dificuldades e facilidades para a realização do acolhimento; e, Equipe atuante no acolhimento.

\section{Concepções sobre o acolhimento}

Nesta categoria foi possível perceber que as enfermeiras consideram o acolhimento como uma escuta inicial das necessidades trazidas pelos usuários. Apesar deste conhecimento, as concepções retratam que, para elas, o acolhimento tem como objetivo triar os usuários, sem necessariamente a realização de uma escuta ampliada. Em alguns momentos, observou-se que, algumas enfermeiras, consideram o acolhimento como uma consulta de enfermagem, sendo realizada previamente à consulta médica. Esse entendimento ficou claramente explícito nas falas a seguir:

Todos os acolhimentos que eu fiz eu sempre passava para o doutor. Todos, por mais que eu soubesse que era uma sessão de agendamento. (E1)

[...] na hora de prestar o acolhimento: escutava a queixa, fazia o exame físico e passava pontualmente o que era necessário. Eu raramente liberava o paciente por conta. (E1)

No acolhimento tu és a primeira pessoa com a qual o doente se expõe, então tu consegues identificar ali através dos sinais, através de uma breve conversa, todo o histórico dele de saúde atual, fazendo um levantamento rapidamente. (...) ou tu passa para o médico, ou agenda uma consulta ou já resolve o problema na hora. (E7) 
A triagem, na real, é uma consulta de enfermagem, uma consulta clínica, onde todos os sinais são vistos e depois passamos para o médico o que a gente percebeu e retornamos para o paciente. (E5)

Através desses depoimentos fica evidenciado que o acolhimento é compreendido como um momento de triagem para organizar o serviço médico. No entanto, o ato de acolher deve ir além, pois a APS para ser resolutiva, deve ter tanto capacidade ampliada de escuta quanto a um sentido ampliado de ofertas para lidar com a complexidade de sofrimentos, adoecimentos, demandas e necessidades de saúde às quais as equipes estão constantemente expostas (Alvarenga et al., 2021).

É importante salientar que no ato de acolher sempre deverá prevalecer o compromisso em realizar a melhor escuta possível das queixas ali relatadas pelo usuário que vem procurar o serviço. O acolhimento não deve ser relacionado a uma prática tecnicista, apenas com verificação de sinais e sintomas, pois o acolhimento vai, além disso. É necessário que a equipe tenha comprometimento e esteja devidamente capacitada para receber e decodificar tais queixas para atender de forma qualificada os que a procuram (Silva \& David, 2021).

\section{Dificuldades e facilidades para a realização do acolhimento}

No processo de acolher, as enfermeiras reconhecem a ESF como um fator facilitador no acolhimento, tendo a visita domiciliar como uma ferramenta capaz de promover ações de saúde à população, garantindo o acesso a quem não pode ir até a unidade. Entretanto, destacam como fatores que dificultam o acolhimento a estrutura física da unidade e a aceitação/entendimento dos usuários em relação ao modo como é realizada esta prática. As mesmas relataram que a falta de salas acaba interferindo diretamente na qualidade do atendimento ao usuário, tanto pela falta de privacidade, quanto pelo fato de prejudicar a concentração do próprio profissional durante a realização do atendimento.

Como nossas salas são ali na frente, dificulta até mesmo a privacidade do paciente. (E4)

A demanda é muito grande, os postos da redondeza negam atendimento, daí se a gente negar Deus me livre. Não temos salas suficientes. Hoje até foi tranquilo, tínhamos duas salas disponíveis, mas quando não tem o paciente chega a esperar até 1 hora por atendimento. (E6)

Por exemplo: dificulta quando chego para chamar a primeira ficha e vejo vinte ali aguardando acolhimento, e sei que todas aquelas pessoas estão necessitando atendimento e tu tens que fazer um bom atendimento com aquela pessoa e tens mais 19 e o tempo está passando. Eu acho que o tempo e a falta de salas. Talvez se tivéssemos mais salas disponíveis para o acolhimento. Essa estrutura pequena dificulta mesmo, porque se a UBS tivesse uma estrutura maior acolheríamos mais pessoas. (E7)

No momento em que as enfermeiras apresentam como fator de dificuldade a falta de salas, nos leva a refletir sobre a demora do atendimento e consequentemente na redução de tempo que elas terão para planejar as ações a serem desenvolvidas através da demanda trazida pelo paciente. Muitas vezes, acabam permitindo um acolhimento mais mecânico que diminui o tempo da escuta, na tentativa de conseguir atender a todos que procuram os serviços de saúde (Doracci \& Lorenzi, 2021).

De acordo com a problemática do espaço físico nas unidades colocado em pauta unanimemente pelas enfermeiras, a PNH destaca que a compatibilização da oferta, a demanda das ações de saúde e a adequação da área física são importantes ferramentas para que haja produção do acolhimento. De acordo com esta política: 
É importante que as unidades de atenção básica tenham estrutura física e ambiência adequadas, como sala de espera (para que os usuários possam aguardar confortavelmente, atenuando seus sofrimentos), sala de acolhimento multiprofissional (para realização do acolhimento individual da demanda espontânea, por meio da escuta qualificada), consultórios (para qualificar as condições da escuta e respeitar a intimidade dos pacientes) e sala de observação (para permitir o adequado manejo de algumas situações mais críticas ou que requerem período maior de intervenção ou acompanhamento) (Brasil, 2010, online).

No que diz respeito ao entendimento errôneo dos usuários sobre o funcionamento do acolhimento, as enfermeiras também destacam que mesmo já tendo explicado aos usuários qual é o intuito e o objetivo do acolhimento, eles continuam a questionar e não entendem a maneira como ele é operacionalizado, note:

[...] a gente já explicou, já fizemos reuniões no CTG, levamos o fluxograma com o protocolo de Manchester, [...] Já fizemos sala de espera, explicando como funciona, mas mesmo assim, são sempre as mesmas pessoas que estão no acolhimento. (E8)

[...] fazemos a sala de espera para explicar o funcionamento do acolhimento, fizemos cartazes, folders, mas a população tem aquela cultura de que: "Estou doente e tu precisa fazer alguma coisa por mim agora. (E2)

Alguns compreendem, mas na sua maioria as pessoas não querem entender. É que a minha queixa e minha dor é sempre maior que a do outro e eu quero ser atendido no momento. Às vezes reclamam: "Eu vi aquela pessoa passando para ser atendida e não fui ainda". Daí eu digo: "Sim. Mas ele era prioridade. Pode aguardar". Na maioria das vezes eles não entendem. (E3)

Ao ser questionada sobre a existência de dificuldades no cotidiano para a realização do acolhimento na APS, apenas uma enfermeira não considerou nenhum fator como relevante, observe: "Não, é que a minha experiência ela é boa entendeu? Não tem o que falar" (E1).

Apesar das dificuldades superarem os fatores contribuintes, a maioria das enfermeiras reconhecem como facilitador a implementação do acolhimento em unidades com ESF, visto que a comunidade se sente mais acolhida pela unidade, garantindo seu acesso aos serviços de saúde. Todas essas questões se refletem nas falas a seguir:

Acho que o pessoal, eles se sentem mais acolhidos, eles sabem que se o posto puder ajudar, o posto vai ajudar, então eles procuram bastante aqui a unidade. (E4)

A equipe contribui. (E2)

Eu acho que a estratégia ela faz muito bem para a população, porque se fosse como antigamente não iríamos na casa. Eu acho que foi bem bom assim, porque a tradicional era só a demanda e o paciente tinha que vir na unidade.

Na verdade, para facilitar, a gente usa a classificação de risco que é o protocolo de Manchester. É a única coisa que vemos que nos ajuda. (E6) 
Considerando as dificuldades e os entraves vivenciados tanto pelas equipes, como pelos usuários, no que tange ao acolhimento, entende-se que faz-se indispensável a amplicação das competências técnicas científicas dos profissionais, para que, de fato seja compreendido o verdadeiro papel de uma escuta efetiva e resolutiva aos pares envolvidos. Permitindo e corroborando com a expanção dos cuidados primários em saúde e como conseguinte com a qualidade de vidad da população adstrita (Camargo \& Castanheira, 2020; Valério et al., 2021).

\section{Equipe atuante no acolhimento}

As enfermeiras consideram que todos da equipe de saúde realizam o ato de acolher ou pelo menos deveriam estar habilitados para esta realização, inclusive a recepção que basicamente é o primeiro contato que os usuários têm com o serviço. Tais afirmativas podem ser percebidas a seguir:

O acolhimento a princípio é realizado pela recepção. [...] isso é realizado pela recepção. Nós avaliamos o que é necessário ser visto hoje e o que pode ser agendado para uma consulta. (E2)

Toda a equipe tem que estar habilitada para fazer o acolhimento. (E2)

De tarde é a enfermagem que faz o acolhimento, de manhã os médicos. Na verdade, uma médica específica que atende junto aos alunos 16 fichas. E de tarde os enfermeiros que classificam com Manchester e passam para os médicos de acordo. (E6)

Todos eles. Geralmente aqui quem acolhe são as enfermeiras, e acredito que todas elas estão muito bem habilitadas. (E7)

De acordo com o MS (Nunes et al., 2021), geralmente o contato inicial dos usuários que procuram os serviços de saúde se dá por meio dos profissionais da recepção, que na maioria das vezes não tiveram nenhuma formação específica para atuar na área da saúde. Assim sendo, é necessário que esses profissionais sejam igualmente envolvidos e estimulados a participarem das reuniões de equipe e, sobretudo, realizarem capacitações que tragam como objetivo a organização do sistema. Aprofundar conhecimentos sobre questões de escuta, no que tange ao resgate da humanização, abordagem de como "receber bem" os usuários, para que seja possível assim, difundir o pensamento centrado somente para a doença e focado especificamente na queixa e, dessa forma ampliar a compreenssão acerca da singularidade dos sujeitos e seus determinantes. Pois quando os usuários se sentem acolhidos, criam vínculos e passam a confiar e recorrer aos profissionais da unidade (Pinto et al., 2021).

Ainda com relação à equipe atuante, procurou-se ir mais além e identificar quais são os profissionais que fazem a linha de frente do acolhimento na unidade de saúde em que cada uma delas atua. Na maioria das unidades elas reconhecem o profissional enfermeiro frente ao acolhimento. Entretanto, não desconsideram o profissional médico, nem tampouco diminuem os profissionais da recepção:

No momento, o acolhimento é feito pelos alunos da medicina do quinto ano. Até então era a enfermagem. (E4)

Enfermagem e de manhã é a médica. Por um entendimento nosso de equipe, mas acredito que todos estão capacitados para isso. (E7)

A recepção e os enfermeiros. (E2) 
A partir desse momento, nota-se que todos os profissionais têm sua importância dentro da equipe sejam eles específicos da área da saúde, ou não. A valorização do trabalho multiprofissional é uma ferramenta essencial para que o serviço possa oferecer uma assistência integral e resolutiva que esteja de acordo com os princípios do SUS, bem como da PNH (Barreto et al., 2019).

Como limitações do presente estudo, um importante aspecto a ser mencionado refere-se a coleta de dados, já que as entrevistas foram feitas em horário de trabalho das profissionais de enfermagem nas UBS, e isso por sua vez, não interrompeu o fluxo de trabalho das mesmas, sendo que os aprofundamentos e percepções podem ter sido perdidas devido a elevada demanda de trabalho e pelo fluxo contínuo de atividades e atendimentos. No entanto, se esse momento pudesse ser realizado em espaço mais apropriado, as compreensões das relações, poderiam se dar de maneira mais aprofundada.

Como contribuição para a prática entende-se que, o aprofundamento e compreensão das fragilidades e potencialidades, corroboram com os fluxos de trabalho e podem resultar em melhorias nos acolhimentos e formas humanizadas de atendimentos prestados à comunidade. Ser e estar sensível as relações que são construídas no meio laboral de saúde instigam os profissionais a se capacitarem com constância - educação permanente em saúde - e buscar formas e modelos dinâmicos que supram as reais necessidades em suas singularidades dos que carecem de auxílio e acolhimento.

\section{Considerações Finais}

Este estudo possibilitou identificar a compreensão das enfermeiras sobre o acolhimento que acontece dentro das unidades as quais elas trabalham, conhecer as dificuldades e facilidades do desenvolvimento desse acolhimento junto a ESF e à população. Ficou evidente que apesar de haver conhecimento por parte das entrevistadas, elas não conseguem identificar e organizar o acolhimento conforme preconizado no seu cotidiano. As dificuldades citadas por elas, prejudicam o atendimento e a escuta ao usuário. Desta forma, em sua maioria, elas não conseguiram encontrar muitos elementos facilitadores que suprissem essas dificuldades e revelam que a realização do acolhimento ainda está em construção, ou seja, precisa ser melhorada e (re)formulada.

Para que esse acolhimento seja qualificado e resolutivo, os profissionais terão que buscar na teoria e na prática as melhores maneiras de aplicá-lo, tais como escuta qualificada e ações programáticas que supram a demanda reprimida, para que assim seja possível superar as dificuldades que as enfermeiras acreditam interromper esse processo. Tornar a prática do acolhimento um fazer humanizado, realizar uma escuta cuidadosa e ao mesmo tempo ampliada, valorizar a subjetividade de cada usuário e certificar o direito a um atendimento digno e adequado dos mesmos.

De modo a permitir maiores aprofundamentos acerca da temática estudada, bem como a percepção de outros cenários, sugerem-se novos estudos de abordagens similares, afim de identificar as fragilidades e as potencialidades existentes, permitindo e construção de novas e inovadoras formas de contribuir para essa realidade e seus pares envolvidos.

\section{Referências}

Alvarenga, J. P. O. et al., (2021). Training models for primary health care: evidence in the context of nursing education. Enferm Foco, 12 (Supl.1), $42-8$.

Báfica, A. C. et al., (2021). Atenção primária à saúde abrangente: ampliando acesso para uma enfermagem forte e resolutiva. Enferm Foco, 12 (Supl.1), 61-6.

Bardin L. (2011). Análise de Conteúdo. (4a ed.), Edições 70.

Barreto, A. C. O. et al., (2019). Perception of the Primary Care multiprofessional team on health education. Rev Bras Enferm, 72 (Supl. 1), $278-85$.

Brasil. Lei no 8.080, de 19 de setembro de 1990. (1990). Dispõe sobre as condições para a promoção, proteção e recuperação da saúde, a organização e o funcionamento dos serviços correspondentes e dá outras providências. http://www.planalto.gov.br/ccivil_03/leis/18080.htm.

Brasil. Ministério da Saúde. Secretaria de Atenção à Saúde. Núcleo Técnico da Política Nacional de Humanização. (2010). Acolhimento nas práticas de produção de saúde. Ministério da Saúde, Secretária de Atenção à Saúde, Núcleo Técnico da Política Nacional de Humanização. $2^{a}$ ed. Brasília: Editora do Ministério da Saúde. 
Research, Society and Development, v. 11, n. 2, e14511225689, 2022

(CC BY 4.0) | ISSN 2525-3409 | DOI: http://dx.doi.org/10.33448/rsd-v11i2.25689

Brasil. Portaria $\mathrm{n}^{\circ}$ 2.436, de 21 de setembro de 2017. (2017). Política Nacional de Atenção Básica. https://bvsms.saude.gov.br/bvs/saudelegis/gm/2017/prt2436_22_09_2017.html.

Brasil. Portaria de Consolidação nº 2, de 28 de setembro de 2017. (2017). Consolidação das normas sobre as políticas nacionais de saúde do Sistema Único de Saúde. https://bvsms.saude.gov.br/bvs/saudelegis/gm/2017/prc0002_03_10_2017.html\#CAPITULOII.

Brasil. Ministério da Saúde. (2013). Acolhimento à demanda espontânea. Secretária de Atenção à Saúde. Departamento de Atenção Básica. $1^{\mathrm{a}}$ ed. Brasília.

Brasil. Ministério da Saúde. (2004). HumanizaSUS - Acolhimento com classificação de risco: um paradigma ético-estético no fazer em saúde. $1^{a}$ ed. Brasília.

Camargo, D. S.; \& Castanheira, E. R. L. (2020). Amplifying access: Team Embracement as a demand management strategy in Primary Health Care (APS). Interface, 24 (05), 1-17.

Doricci, G. C.; \& Lorenzi, C. G. (2021). Co-management in the context of Brazil's National Humanization Policy: an integrative review. Ciênc Saúde Coletiva, 26 (08), 2949-2959.

Gusmão, R. O. M. et al., (2021). Welcoming in primary health care in the perception of the multidisciplinary team. Rev pesq cuid fundam online, 13 (1), 1590 1595 .

Lacerda, M. R.; Ribeiro, R. P.; \& Costenaro, R. G. S. (Org.). (2018). Metodologias da pesquisa para a enfermagem e saúde: da teoria à prática. $2^{\mathrm{a}}$ ed. Porto Alegre: Moriá Editora.

Lopes, O. C. A. et al., (2020). Competences of nurses in the Family health Strategy. Esc Anna Nery, 24 (2), 1-8.

Marques, C. R.; \& Silva, C. T. X. (2020). The difficulties in the implementation of the reception in primary health care: an experience report at the bandeiras family health unit in anápolis-go. Braz J of Develop, 6 (20), 96805-96810.

Minayo, M. C. S. (2014). O desafio do conhecimento: pesquisa qualitativa em saúde. (14a ed.), HUCITEC.

Morelato, C. S. et al., (2021). Receiving spontaneous demand in Primary Care: nurses’ learning needs. Rev Bras Enferm, 74 (2), 1-9.

Nunes, T. B. et al., (2021). Implementation of an improvement cycle in the care of a primary health care. Rev Bras Enferm, 74 (1), 1-7.

Pinto, J. M. et al., (2021). Atribuições da Enfermagem e a Importância do Acolhimento do Enfermeiro na Atenção Básica: Uma Revisão Bibliográfica Integrativa. JNT- Facit Business and Technology Journal, 26 (1), 200-211.

Silva, T. F.; \& David, H. M. S. L. (2021). Acolhimento, Redes sociais e produção do cuidado na Atenção Básica em Saúde no Município do Rio de Janeiro, Brasil. Rev REDES, 32 (1), 67-81.

Silva, T. F.; David, H. M. S. L.; \& Romano, V. F. (2020). Análise do acolhimento a partir das relações na Atenção Básica no município do Rio de Janeiro. Rev Bras Med Fam Comunidade, 15 (42), 2326.

Valério, F. C. E. P. et al., (2021). Advances and challenges for the implementation of the user embracement in the Family Health Strategy. Braz J of Dev, 7 (7), 68875-68890. 